\title{
Fault Tolerance in Hyperbus and Hypercube Multiprocessors Using Partitioning Scheme
}

\author{
Shih-Chang Wang and Sy-Yen Kuo \\ Department of Electrical Engineering \\ National Taiwan University \\ Taipei, Taiwan, R.O.C. \\ sykuo@cc.ee.ntu.edu.tw
}

\begin{abstract}
In this paper, the partitioning scheme is used to achieve fault tolerance in hyperbus and hypercube multiprocessors. Unlike other schemes, processor faults are assumed to be randomly distributed. We propose a novel and practical load redistribution method to tolerate processor faults in a hyperbus structure with insignificant overhead (a slowdown of 2 for computation and a slowdown of 3 for communication in the worst case). Standard routing and broadcasting algorithms were implemented on hypercube computers. To achieve fault tolerance, we present routing and broadcasting algorithms for a faulty hypercube with at most n-1 faults. Compared with other existing algorithms, our methods have better performance in most measures.
\end{abstract}

\section{Introduction}

The boolean $n$-dimensional hypercube ( $n$-cube) computer is an interconnection with $N=2^{n}$ processors. The $n$-cube computer can be viewed as having processors(nodes) placed at the corners of an $n$-dimensional cube, and each edge connected to two processors. Processors in an $n$-cube communicate by passing messages. Extensive research has been done on hypercubes, and many commercial hypercube computers have successfully been implemented The topological properties of a hypercube were introduced in $[3,4]$ and various other topologies can be mapped into a hypercube. The hypercube structure can handle a reasonable message traffic, and has some degree of fault tolerance. Many issues of fault tolerance in a faulty $n$-cube have been proposed [6-7, 9].

The generalized hyperbus structure $(G H B)$ were proposed in [5] and compared to other parallel computers. In addition, the properties of the $G H B$ were investigated and presented in [5]. The hyperbus structure is obtained

Acknowledgment: This research was supported by the National Science Council, Taiwan, R.O.C., under Grant NSC 83-0408-E002-022. from $G H B$ by assuming that there are only two buses in each dimension. One of the advantages of the hyperbus structure is that each processor contains only two $\mathrm{I} / \mathrm{O}$ ports. This structure is extremely suitable for local area computer networks.

Several researchers have examined the partitioning scheme in hypercubes $[1,6,8]$. The partitioning scheme is attractive because it can make complex problems simple. An $m$-partition of an $n$-cube is to partition the $n$ cube into several $m$-cubes. Let $F$ be the set of faulty processors in an $n$-cube. We say that an $m$-cube $S$ tolerates $F$ iff $S$ contains a connected component of at least $2^{m-1}+1$ nodes and all of them are healthy. We will say that an $m$-partition $P$ tolerates $F$ iff for every $m$-cube $S$ in $P, S$ tolerates $F$ [1]. A fault tolerant $m$-partition of an $n$-cube is important because it guarantees that for any pair of adjacent $m$-cubes there is at least one edge connecting the largest healthy connected components in the $m$-cubes. If we tolerate processor faults for one application in each $m$-cube of an $m$-partition, we can implement the application with an insignificant efficiency slowdown in a faulty $n$-cube. The efficiency slowdown of an implementation for an application on a faulty hypercube is defined to be the time of the application required on the faulty hypercube divided by the time required on the fault-free hypercube.

The partitioning scheme can also be applied to the hyperbus. If the number of processor faults in a hyperbus structure is in the tolerant range, then we use the partitioning scheme to propose a constructive approach which finds healthy processors to simulate faulty processors. We show that any job designed for the faultfree hyperbus structure can be implemented on a faulty hyperbus structure in which the number of "special buses" generated by the faulty processors is at most $n$ with a slowdown of 2 for computation and a slowdown of 3 for communication in the worst case.

Several researchers have given methods for faulttolerant routing [11-12, 14-15] and broadcasting[11-13, 1516] algorithms in faulty hypercubes. Using the partitioning scheme, we develop simple routing and broadcasting algorithms in faulty hypercubes. Compared with existing algorithms, our methods have better 
performance in most measures. In section 2, we give the assumptions and definitions for our work. Fault tolerance in hyperbus structures is addressed in section 3. Sections 4 and 5 present routing and broadcasting in faulty hypercubes, respectively. Discussions and comparisons are given in section 6 followed by the conclusions in section 7 .

\section{Preliminaries}

First, we make several assumptions which are commonly made in the literature: 1) Only processor faults are considered. In fact, link faults in a faulty hypercube can be tolerated by viewing the two end-nodes of the faulty link as "faulty" nodes. 2) All processor faults are static and detectable. 3) A faulty processor can not compute and can not send messages. 4) The messages sent to a faulty processor will be lost. Next, some definitions and notations are introduced[1]. A multiset is a collection of objects in which repetitions are allowed. Let $T$ be a multiset and $X$ be an element in $T$. The notation $\operatorname{mult}(X, T)$ is the number of times $X$ appears in $T$, and $\operatorname{mult}(T)$ is the maximum number of mult $(X, T)$, for all $X \in T$.

Each node in an $n$-cube is labeled with an $n$ dimensional binary vector $\left(x_{1} x_{2} \ldots x_{n-1} x_{n}\right)$, where $x_{\mathrm{i}} \in\{0,1\}$, for $1 \leq \mathrm{i} \leq n$. The dimension associated with $x_{\mathrm{i}}$ is called the $i$ th dimension. The neighboring node of a node $X$ is different from $X$ in a single dimension of their binary representations. The Hamming distance between two nodes $u$ and $v$ in an n-cube is denoted by $H(u, v)=\|u \oplus v\|$. Symbol $\oplus$ denotes the bitwise exclusive-OR $(X O R)$ operation on binary numbers.

An $m$-cube of an $n$-cube is denoted by $M=\left(m_{1}\right.$ $\left.m_{2} \ldots m_{n}\right)$, where exactly $m$ of the $m_{\mathrm{i}}$ 's are *'s(don't cares) and the remaining $m_{\mathrm{i}}$ 's are either 0's or 1's. Let the set $P \subseteq\{1,2, \ldots, \mathrm{n}\}$ and $|P|=m$. An $m$-partition $P$ contains $2^{n-}$ $m m$-cubes where an $m$-cube $M$ is in $P$ iff for all i, $1 \leq \mathrm{i} \leq n, m_{\mathrm{i}}=*$ iff $\mathrm{i} \in P$. Given the multiset of $n$ dimensional binary vectors $T$ and an $m$-partition $P$ of an $n$-cube, the notation $\operatorname{proj}(P, T)$ is the multiset of $(n-m)$ dimensional binary vectors obtained by removing the $m$ dimensions specified by $P$ for each vector in $T$. The notation @ $(P, T)$ denotes the value of $\operatorname{mult}(\operatorname{proj}(P, T))$.

For example: Let $T=\{(1100),(1011),(0001)\}$ and $P=\{2\}, \operatorname{proj}(P, T)=\{(100),(111),(001)\}$ and $@(P, T)=1$.

\section{Fault Tolerance in Hyperbus Structures}

The generalized hyperbus structure $(G H B)[5]$ consists of $N$ buses with $N=m_{n} \times m_{n-1} \times \ldots \times m_{1}$. Let $N=2^{n}$, and $m_{\mathrm{i}}=2$ for $1 \leq \mathrm{i} \leq n$, we obtain the hyperbus structure. A bus in the hyperbus structure is denoted by an $n$-tuple $\left(x_{1} x_{2} \ldots x_{n}\right)$ with $x_{\mathrm{i}} \in\{0,1\}$ and $1 \leq \mathrm{i} \leq n$. A processor in the hyperbus structure is denoted by $\left(x_{1} x_{2} \ldots x_{\mathrm{i}-1}\right.$ [ $\left.\left.y_{\mathrm{i}} z_{\mathrm{i}}\right] x_{\mathrm{i}+1} \ldots x_{n}\right)$, i.e., with $x_{\mathrm{i}}$ replaced by a 2 -tuple $\left[y_{\mathrm{i}} z_{\mathrm{i}}\right]$ and $y_{\mathrm{i}}, z_{\mathrm{i}} \in[0,1\}$. This means that the processor is connected to the two buses $\left(x_{1} x_{2} \ldots x_{\mathrm{i}-1} y_{\mathrm{i}} x_{\mathrm{i}+1} \ldots\right.$ $\left.x_{n}\right)$ and $\left(x_{1} x_{2} \ldots z_{\mathrm{i}} x_{\mathrm{i}+1} \ldots x_{n}\right)$. A hyperbus structure with $2^{3}=8$ buses and $N=2 \times 2 \times 2$ is shown in Fig. 1 .

We will use the following lemma in [6] to show our results in Theorem 1.

Lemma 1: For $n \geq 1$, given a set $F$ of $n$ or fewer faulty nodes in an $n$-cube, there exists a 1-partition $P$ of the $n$ cube such that @ $(P, F) \leq 1$.

We can view a hyperbus structure with $2^{n}$ buses as an $n$-cube structure and assume that a bus can be in one of the following two states: "special bus" or "normal bus". If a processor in a hyperbus structure is faulty, the buses which are connected to the faulty processor are special buses, otherwise they are normal buses. In a hyperbus structure, each processor is connected to two buses and hence a faulty processor will generate two special buses. For example, in Fig. 1, if processor $(00[01])$ and processor (0[01]1) are faulty, the buses $(000),(001)$, and (011) are special buses, and the rest are normal buses.

We assume that a bus can be held by only one pair of processors which can communicate through the bus in a single time step. Because a processor in a hyperbus structure has two $\mathrm{I} / \mathrm{O}$ ports, we assume that a processor can process two I/O operations in one time step.
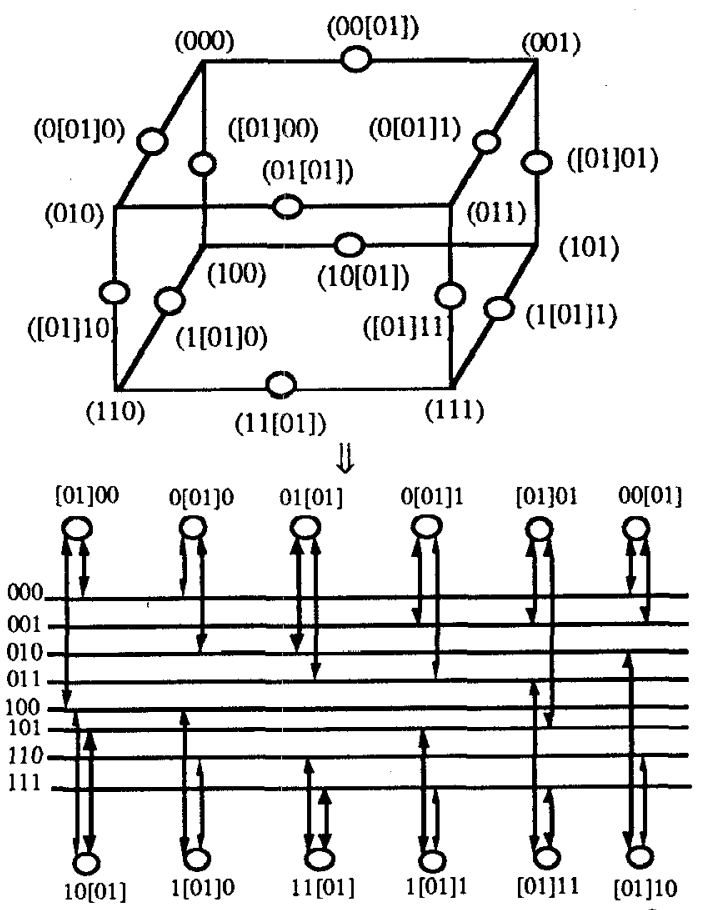

Fig. 1. A hyperbus structure with $2^{3}=8$ buses and $N=2 \times 2 \times 2$. 
Theorem 1: For $n \geq 1$, given a faulty hyperbus structure with $2^{n}$ buses and a set $F$ of faulty processors, if the number of special buses generated by the fault set $F$ is less than or equal to $n$, then any job on the faulty hyperbus structure can be implemented with, in the worst case, a factor of 2 slowdown for computation, and a factor of 3 slowdown for communication.

Proof: Let the set of special buses generated by the fault set $F$ be denoted by $F^{\prime}$. Since $\left|F^{\prime \prime}\right| \leq n$, we know from Lemma 1 that there exists a 1-partition $P$ of the faulty hyperbus structure such that each 1-cube in $P$ contains at most one special bus. We only need to consider pairs of adjacent 1-cubes as in Fig. 2 where $b_{i}$ and $p_{i}$ denote a bus and a processor, respectively.

For any pair of adjacent 1-cubes $\{A, B\}$ in $\mathrm{P}$ (see Fig. $2)$, because each 1-cube contains at most one special bus, the faulty node must be located on the link which connects two 1-cubes in $P$. By symmetry, we can assume that the faulty processor is $p 1$ without loss of generality. Then $b 1$ and $b 2$ are special buses; $b 3$ and $b 4$ are normal buses. The computation on the faulty processor is taken over by the processor which connects to the same pair of 1 -cubes as the faulty processor. For example, in Fig. 2, the workload of processor $p 1$ is taken by processor $p 3$. Because each healthy processor is responsible for at most 1 faulty node, all computations can be performed with at most a factor of 2 slowdown.

Each communication operation is implemented in three steps as described below. Sending messages between healthy nodes is performed in the first time step. Sending messages from or to a faulty processor starts at the beginning of the second time step, and take the following new paths. The notation $(p i \stackrel{b j}{\longrightarrow} p k)$ denotes that processor $p i$ sends a message to the processor $p k$ through bus $b j$ in one time step.

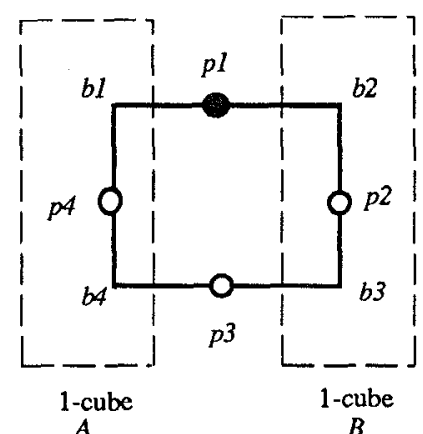

Fig. 2. A pair of adjacent 1-cubes with one faulty node(black).

\footnotetext{
The path $(p 1 \stackrel{b 2}{\longrightarrow} *)(*$ denotes a healthy processor and $p 1$ is faulty) is replaced by the path $(p 3 \stackrel{b 3}{\longrightarrow} p 2 \stackrel{b 2}{\longrightarrow})$. The path $(p 1 \stackrel{b 1}{\longrightarrow} *)$ is
}

replaced by the path $(p 3 \stackrel{b 4}{\longrightarrow} p 4 \stackrel{b 1}{\longrightarrow} *)$. The path $(* \stackrel{b 1}{\longrightarrow} p 1)$ is replaced by the path ( * $\stackrel{b 1}{\longrightarrow} p 4 \stackrel{b 4}{\longrightarrow} p 3)$. The path $(* \stackrel{b 2}{\longrightarrow} p 1)$ is replaced by the path $(* \stackrel{b 2}{\longrightarrow} p 2 \stackrel{b 3}{\longrightarrow} p 3)$.

It is straightforward to verify that no processors execute more than two I/O operations and no bus conflicts exist in any single time step.

Theorem 1 provides a constructive approach in finding healthy processors to take over the work of faulty processors. In the worst case, the size of the set $F$ is at most $\left\lfloor\frac{n}{2}\right\rfloor$. In the best case, the size of $F$ is at most $n-1$. An example for Theorem 1 is shown in Fig. 3. The special buses are 000,001 , and 011 . There is at most one special bus in any 1-cube. The work of the faulty node $00[01]$ is performed by node $10[01]$, and the work of the faulty node $0[01] 1$ by node 1[01]1. In addition, communication for each faulty node is completed as previously described. In the next two sections, we will demonstrate how the partitioning scheme can be used to implement communication algorithms in a faulty $n$-cube.

\section{Routing in a Faulty Hypercube}

The standard routing algorithm for a fault-free $n$-cube is described in [2]. This algorithm, which we call ROUTE, is shown in Fig. 4. Let curr denote the current node and dest denote the destination node. ROUTE sends a message through the link whose label corresponds to the first bit position in which the curr and dest node labels differ. This simple algorithm always finds a minimum length path. This length is at most $n$, and the computation overhead of the routing algorithm is very small. The algorithm ROUTE has been implemented on hypercube computers using various approaches. In the algorithm ROUTE, determining a next link for sending one message needs $\mathrm{O}(n)$ bit-comparison time, where $n$ is the number of dimensions in the $n$-cube.

However, algorithm ROUTE does not work if the hypercube is faulty. We will apply the partitioning scheme in a faulty hypercube with at most $n-1$ faulty nodes(processors) to implement routing. Our a:gorithm has computation time overhead with $\mathrm{O}(n)$ bit-comparison time. This algorithm is easy to be implemented in a faulty hypercube. We also assume that there exists a mechanism as in [9] such that any healthy node in an $n$ cube can determine the faulty/nonfaulty status of its neighboring nodes, and each processor has a fault list for recording the status of neighboring nodes. Therefore, we need additional $\mathrm{O}(n)$ bits space for each processor. To facilitate our discussion, we need some more notations and definitions. The dimensions which are local to the 2cubes in a 2-partition are called the internal dimensions, otherwise they are called the external dimensions. The 
neighboring nodes of a node $W$ within the same 2-cube are called the buddies of the node $W$.

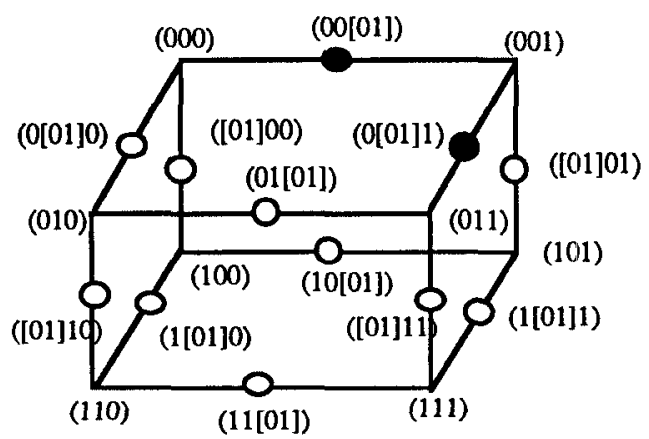

(a)

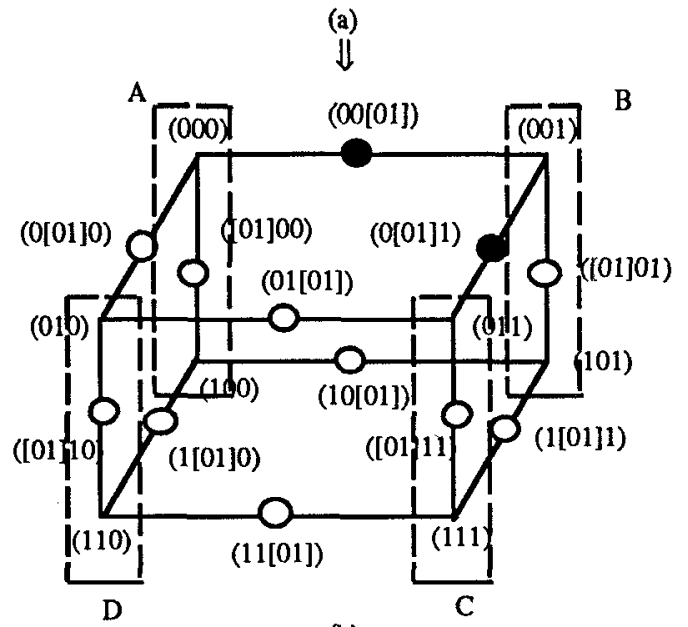

(b)

Fig. 3. (a) Two faulty nodes ( 00[01] ) and (0[01]1). (b) Use a 1-partition $P=\{1\}$ to partition the 3-cube into four 1-cubes, $A$, $B, C$, and D.

Algorithm ROUTE

\{ curr is the current node and dest is the destination node. $\mathrm{f}_{1}$ (curr,dest) is the first dimension from left that the two labels curr and dest differ.\}

begin

for every message

if curr $=$ dest

then Retain message in node curr;

else Send message to neighboring processor via link $\mathrm{f}_{1}$ (curr, dest).

end

\section{Fig. 4. Algorithm ROUTE for node-to-node routing in a fault-free hypercube.}

For example, in Fig. 5, a 4-cube is partitioned into four 2 -cubes $(* * 00),(* * 01),(* * 10)$, and $(* * 11)$ using a 2 partition $P=\{1,2\}$. The $1 s t$ and 2 nd dimensions from left are the internal dimensions, and the $3 r d$ and 4 th dimensions are the external dimensions. The buddies of node (1110) are nodes (0110) and (1010). In a 2-partition, each node has only two buddies. The function $\mathrm{f}_{\mathrm{i}}\left(x_{k}, x_{j}\right)$ is the position of the $i$ th external bit from left, that is, the $i$ th external dimension, at which the labels $x_{k}$ and $x_{j}$ differ. The function $\mathrm{gi}_{\mathrm{i}}\left(x_{k}, x_{j}\right)$ is the position of the $i$ th internal bit from left, that is, the ith internal dimension, at which the labels $x_{k}$ and $x_{j}$ differ. The function $h_{\mathrm{i}}$ (curr) lets the current node curr check the status of its neighboring node in the $i$ th dimension. If the neighboring node in the $i$ th dimension is not faulty, then $h_{i}$ (curr) returns TRUE, else returns FALSE. If there is no value $i$ for $\mathrm{f}_{\mathrm{i}} \mathrm{O}$ or $\mathrm{g}_{\mathrm{i}} \mathrm{O}$, then $\mathrm{f}_{\mathrm{i}} \mathrm{O}$ or $\mathrm{g}_{\mathrm{i}} \mathrm{O}$ is assumed to have the value 0 . For example, in Fig. 5:

$$
\begin{array}{ll}
\mathrm{f}_{1}(0100,1100)=0 & \mathrm{f}_{2}(0100,0111)=4 \\
\mathrm{~g}_{1}(0100,1100)=1 & \mathrm{f}_{1}(0100,0001)=4 \\
\mathrm{f}_{2}(0100,0001)=0 & \mathrm{~h}_{1}(1000)=\text { FALSE } \\
\mathrm{h}_{2}(1000)=\text { TRUE } &
\end{array}
$$

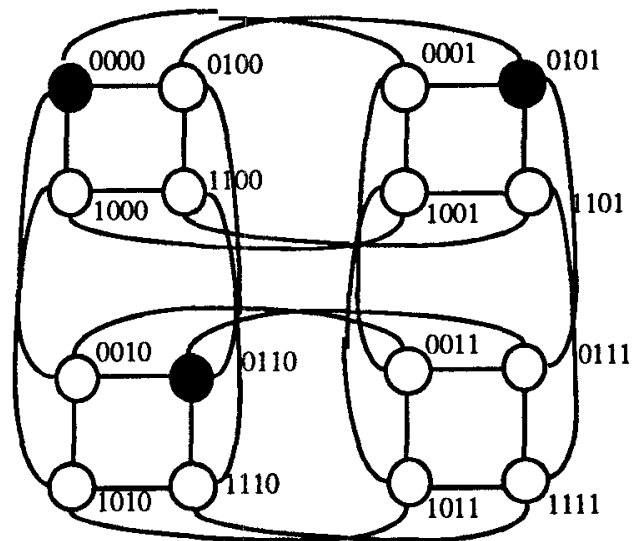

Fig. 5. A 4-cube which is partitioned into four 2-cubes using a 2-partition $P=\{1,2\}$.

We will need the following lemma in [1] for the development of our algorithm, and its complexity is $\mathrm{O}(n)$.

Lemma 2: For $n \geq 2$, given a set $F$ of $n-1$ or fewer faulty nodes in an $n$-cube, there exists a 2-partition $P$ of the $n$-cube such that @ $(P, F) \leq 1$.

If the number of faulty processors is equal to or greater than $n$ in an $n$-cube, there may exist an isolated healthy processor. Therefore, we will assume that the number of faulty processors in a $n$-cube is at most $n-1$. From Lemma 2, for a set $F$ of at most $n-1$ faulty nodes in an $n$ cube, there exists a 2-partition $P$ such that $@(P, F) \leq 1$, that is, there exists none or one faulty node in any 2-cube in $P$. It denotes that there exists one link with both end nodes being healthy to connect a pair of 2-cubes for any pair of 2-cubes in $P$. So the routing algorithm can be implemented on a faulty $n$-cube. The main idea is to route one message from the 2-cube with the current node to the 2-cube with the destination node. This goal is easy to be accomplished by reducing the differences of the external dimensions between the current node and the destination node. The routing algorithm, F_ROUTING, for a faulty $n$-cube is shown in Fig. 6 . The following 
Lemma 3 and Theorem 2 formally present the above results.

Lemma 3: For $n \geq 2$, given a set $F$ of $n-1$ or fewer faulty nodes in an $n$-cube, there exists a 2-partition $P$ such that any pair of adjacent 2-cubes $X$ and $Y$ in $P$ is different in some dimension $i$. If the neighboring node $y 1$ (in $Y$ ) of the healthy node $x 1$ (in $X$ ) in the $i$ th dimension is faulty(see Fig. 7), there exist some links with both end nodes being healthy, and one of the end nodes is the healthy buddy of node $x l$, and the other is in the 2-cube $Y$.

Proof: From Lemma 2, there exists a 2-partition $P 1$ such that @ $(P 1, F) \leq 1$. Let $P=P 1$. Any pair of adjacent 2 -cubes can be represented as in Fig. 7. The following cases are possible scenarios for a pair of adjacent 2-cubes in $\mathrm{P}$.

1. Neither $X$ nor $Y$ has a faulty node.

2. Only $X$ or $Y$ contains one faulty node.

3. $X$ and $Y$ each contains a faulty node.

By using the symmetry property, it is straightforward to check the three cases to verify Lemma 3.

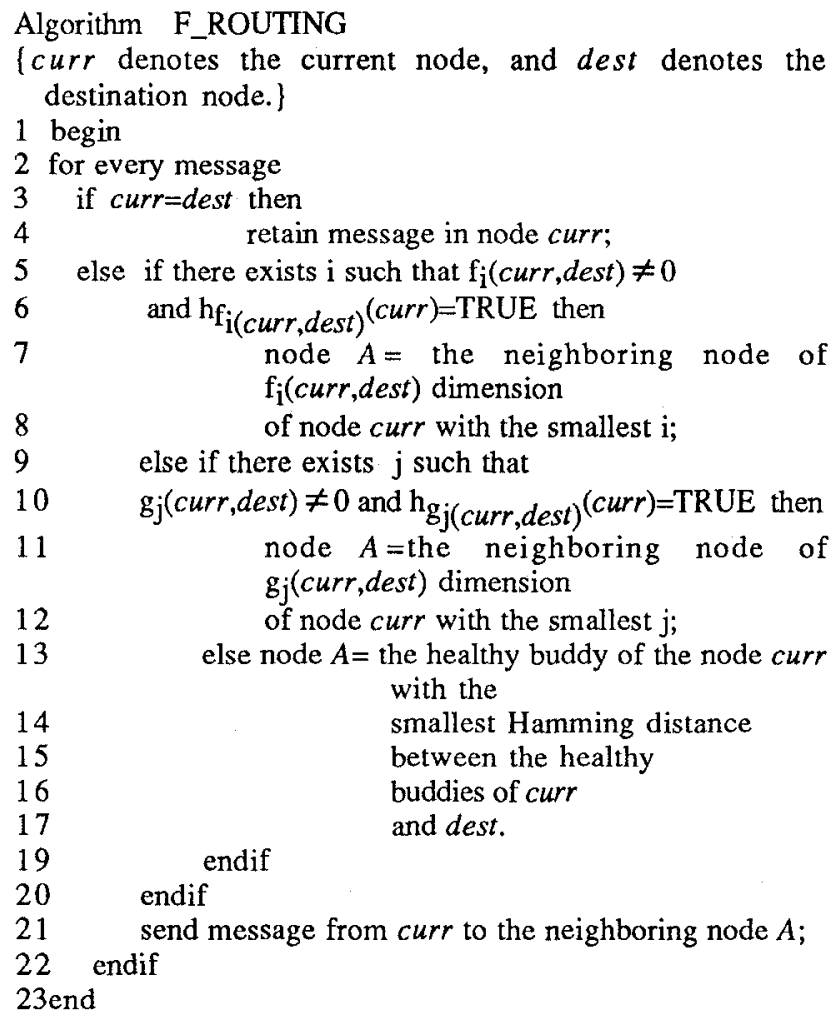

\section{Fig. 6. Routing algorithm F_ROUTING for} an $n$-cube with at most $n-1$ faulty nodes.

Theorem 2: For $n \geq 2$, given a set $F$ of $n-1$ or fewer faulty nodes in an $n$-cube, there exists a 2-partition $P$ such that the algorithm F_ROUTING will find a correct path of finite length to transfer a message.

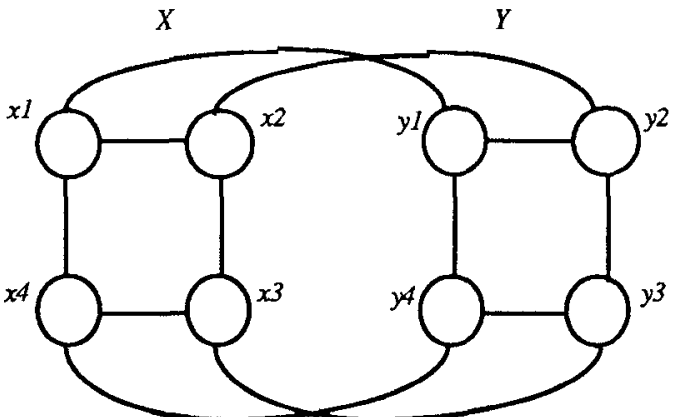

Fig. 7. A pair of adjacent 2-cubes $X$ and $Y$.

Proof: From Lemma 2, there exists a 2-partition $P$ such that $@(P, F) \leq 1$. We use the 2-partition $P$ to partition the faulty $n$-cube. Recall that the time to compute $P$ is $\mathrm{O}(n)$. We can install the information of $P$ in each healthy processor. This action just takes a very small time overhead. Note that there are three possible relationships between the nodes curr and dest in $P$.

The first one is if node labels curr and dest are the same, then node curr retains the message. This is done in line 3 of Fig. 6.

The second one is that the nodes curr and dest have differences in the external dimensions. If the neighboring nodes of node curr in the different external dimensions are not all faulty, then F_ROUTING sends the message to the healthy neighbor which has the smallest different external dimension $i$ from the left. This is done in line 5. If the neighbors of node curr in the different external dimensions are all faulty and because any healthy node has at least one healthy buddy, from Lemma 3, there exists at least one healthy buddy of node curr to reduce the differences in the external dimensions. We select the healthy buddy which can also reduce the difference in internal dimensions if possible. This is done in line 9. If no healthy buddy can reduce the difference in internal dimensions, then we select the healthy buddy $x$ which has the minimum Hamming distance between the nodes $x$ and dest. This is done in line 13. From the above discussion, we know that $F$ ROUTING can send a message from the 2-cube with node curr to the 2-cube with node dest .

The last one is that the nodes curr and dest are local to the same 2-cube. It is straightforward to show that any pair of healthy nodes in a 2-cube of $P$ can exchange a message successfully in line 9 . Obviously, a message can be sent correctly by using algorithm F_ROUTING, and it finds a path of finite length.

The functions $\mathrm{f}_{\mathrm{i}} O$ and $\mathrm{g}_{\mathrm{i}} O$ take $\mathrm{O}(n)$ bit-comparison time. The function $h_{i}()$ and line 13 in Fig. 6 take $O(1)$ time. Therefore, the time complexity of algorithm F_ROUTING is $\mathrm{O}(n)$. The algorithm F_ROUTING takes $\bar{O}(n)$ bit-comparison time to determine a next link for sending one message in each processor of an $n$-cube. $\mathrm{O}(n)$ additional bits is required in each processor for maintaining a fault list. It is easy to see that the 
maximum length for sending a message from $x$ to $y$ is $2 H(x, y)+2$

\section{Broadcasting in a Faulty Hypercube}

The standard algorithm(see Fig. 8.) for broadcasting a message to all nodes from a single source is given in [2]. The number of time steps in the algorithm is $n$, where $n$ is the number of dimensions of the hypercube. Recall that there may exist an isolated healthy processor if the number of faulty processors is greater than $n-1$ in an $n$ cube, so we assume the number of faulty processors is less than $n$. An example for the standard broadcasting algorithm is shown in Fig. 9(The integer $i$ next to a link denotes that the message passing of the link is executed at the $i$ th time step, for $1 \leq i \leq 4$ ).

A straightforward broadcasting algorithm on a faulty $n$ cube with at most $n-1$ faults is described as follows. It uses algorithm BROADCAST in Fig. 8 and the function $\mathrm{h}_{\mathrm{i}}(X)$. Only when CONTROL $[i]=1$ and the neighbor of node curr in the $i$ th dimension is healthy, can node curr send both the message and the modified CONTROL via link $i$. Unfortunately, This method will make some healthy nodes unable to receive the message. For example, let the faulty nodes in Fig. 9 be (1010), (1001) and (1111). Let node (1000) be the source, it is evident reason that node 1011 will not receive the broadcasted message. Such an incomplete broadcasting process is called a loss broadcasting .

We will present a new fault-tolerant broadcasting algorithm that can be implemented on a faulty $n$-cube with at most $n-1$ faults. Let the functions $\mathrm{f}_{\mathrm{i}}(X)$ and $\mathrm{g}_{\mathrm{i}}(X)$ denote the positions of the ith external and internal dimensions from the left for $X$ in $P$, respectively. The function $l(X)$ denotes that the assignment operations executed on $X$ are only for internal dimensions. For example, if $P=\{3,4\}, f_{1}(* * * *)=1, f_{2}(* * * *)=2$,

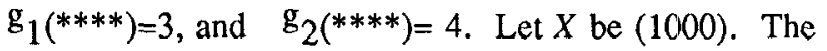
operation $l(X)=(11)$ will result in $X=(1011)$.

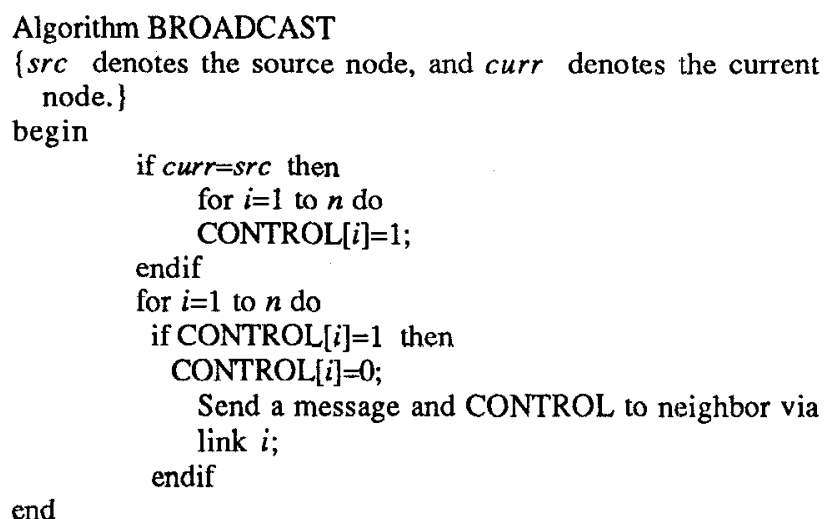

Fig. 8. Algorithm BROADCAST in a faultfree hypercube.

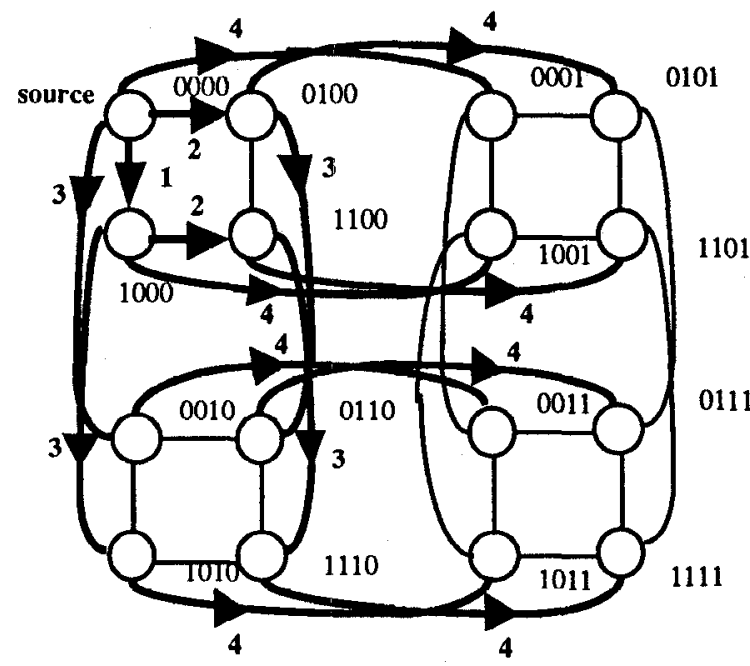

Fig. 9. Broadcasting a message in a faultfree 4-cube using the algorithm BROADCAST.

Theorem 3: For $n \geq 2$, given a set $F$ of $n-1$ or fewer faulty nodes in an $n$-cube, there exists a 2 -partition $P$ such that the algorithm F_BROADCAST(see Fig. 10) can complete the broadcasting on the healthy processors.

Proof: From Lemma 2, there exists a 2-partition $P$ such that @ $(P, F) \leq 1$. We use the 2-partition $P$ to partition the $n$-cube. The $n$-cube can be viewed as an (n2)-cube structure with $2^{n-2} 2$-cubes. Recall that for a pair of adjacent 2-cubes, there is at least one edge connecting the 2-cubes. The links connecting a pair of adjacent 2cubes are in the external dimensions. The 2-cubes of the $n$-cube in $P$ are called $S B$ s (subcube blocks), and we have $2^{n-2} S B$ s in $P$. In the algorithm BROADCAST, each node only receives one message, and the process of the broadcasting is complete(that is, all processors can receive the broadcasting message). In the algorithm BROADCAST, if the $i$ th bit of CONTROL is equal to 1 , then the node curr has to broadcast the message corresponding to the CONTROL to the $i$ th neighboring node. Therefore, the node curr makes the $i$ th dimension of the CONTROL be 0 , and sends the message and the modified vector CONTROL to the $i$ th neighboring node. We can use the same approach as algorithm BROADCAST to implement our CONTROL vector.

Algorithm F_BROADCAST broadcasts the message among those $S B$ s. The same scheme of CONTROL used in BROADCAST is used to implement the external dimensions of CONTROL using in F_BROADCAST. It is clear that each $S B$ of the $n$-cube will correspond to an unique sending $S B$ except the $S B$ with source. Let one healthy node in $S 1$ (is a subcube block) send the message to the other SBs which S1 corresponds to. So, each node only corresponds to one sending node except the source node. F_BROADCAST first broadcasts the message to every $S B \bar{B}$ (lines 9..19). If there are 1 's in the external 
dimensions of CONTROL for node $X$, but the neighbors of $X$ in such external dimensions are all faulty, then from Lemma 3 , the healthy buddy which is not broadcasted can broadcast the message to such $S B \mathrm{~s}$. This goal can be achieved as follows. First, node $X$ sends message and the modified CONTROL to one healthy buddy. Next, $X$ sets its external dimensions of CONTROL to be all 0's and broadcasts continually the message to another buddy if possible(lines 22..30). From above discussions, we know that F_BROADCAST first sends the message to each $S B$ if possible. Notice that the internal dimensions of CONTROL which is sent from one $S B$ to another $S B$ must be all 1 's(This denotes that the message must be broadcasted inside a $S B$ ). If the broadcasting among $S B$ s is finished, then F_BROADCAST begins broadcasting within a $S B$. It is easy to check that lines 22.30 achieve the goal. The proof is completed.

In the worst case, $2 n-2$ time steps are needed to broadcast a message. The time steps for broadcasting among those $S B$ s are at most $2(n-2)$ (if $c u r r$ can not broadcast the message to any neighboring $S B$ s directly), and the time steps for broadcasting within a $S B$ are at most 2. The functions $\mathrm{f}_{\mathrm{i}} \mathrm{O}$ and $\mathrm{h}_{\mathbf{i}} \mathrm{O}$ take $\mathrm{O}(1)$ time. We need $O(n)$ time to determine a next link for broadcasting a message. $\mathrm{O}(n)$ bits are required in each processor for maintaining a fault list. If the $n$-cube is nonfaulty, then just $n$ time steps are required by F_BROADCAST.

\section{Discussions and Comparisons}

In this section, we compare the algorithms F_ROUTING and F_BROADCAST with existing faulttolerant algorithms in the literature and give discussions.

\subsection{Performance Measures}

The performance measures examined are:

》Time Complexity for Preprocessing, $T_{p}$, which is the time overhead needed before fault-tolerant communications. For example, the preprocessing work in our approach is to determine a fault-tolerant 2-partition.

»Time Complexity for Each Node, $T_{n}$, which is the time that each node takes in determining the next intermediate node for a received message.

》 Maximum Number of Lengths/Steps, $L_{m}$, which is the lengths/steps in the worst case to complete a routing/broadcasting.

»Message Size, $M_{S}$, which is the size of message for sending data. A message may contain data field and other control fields.

》Fault Type, $F_{k}$, which is the faults that can be tolerated in a fault-tolerant scheme.

»Range of Faults, $R_{f}$, which is the upper bound on the number of faults that the algorithm is guaranteed to work correctly.

\subsection{Discussions}

The algorithms F_ROUTING and F_BROADCASTING are also suitable for link faults with some modifications. First, we can view the two end-nodes of a faulty link as "faulty" nodes and then determine the faulttolerant 2-partition for faulty nodes. Second, we let the end-nodes of a faulty link return to their real states (that is, if the end-node is healthy, it is the nonfaulty node.) when our algorithms are used. For a nonfaulty node $G$, if the link which connects $G$ with its healthy neighbor $U$ (in the $i$ th dimension) is faulty, the function $\mathrm{h}_{\mathrm{i}}$ ) for $G$ and $U$ will be viewed as FALSE in our algorithms. With the above modifications, it is clear that our algorithms are also suitable for link faults. These modifications need at most $n$ bits to record the states of connecting links for a nonfaulty node.

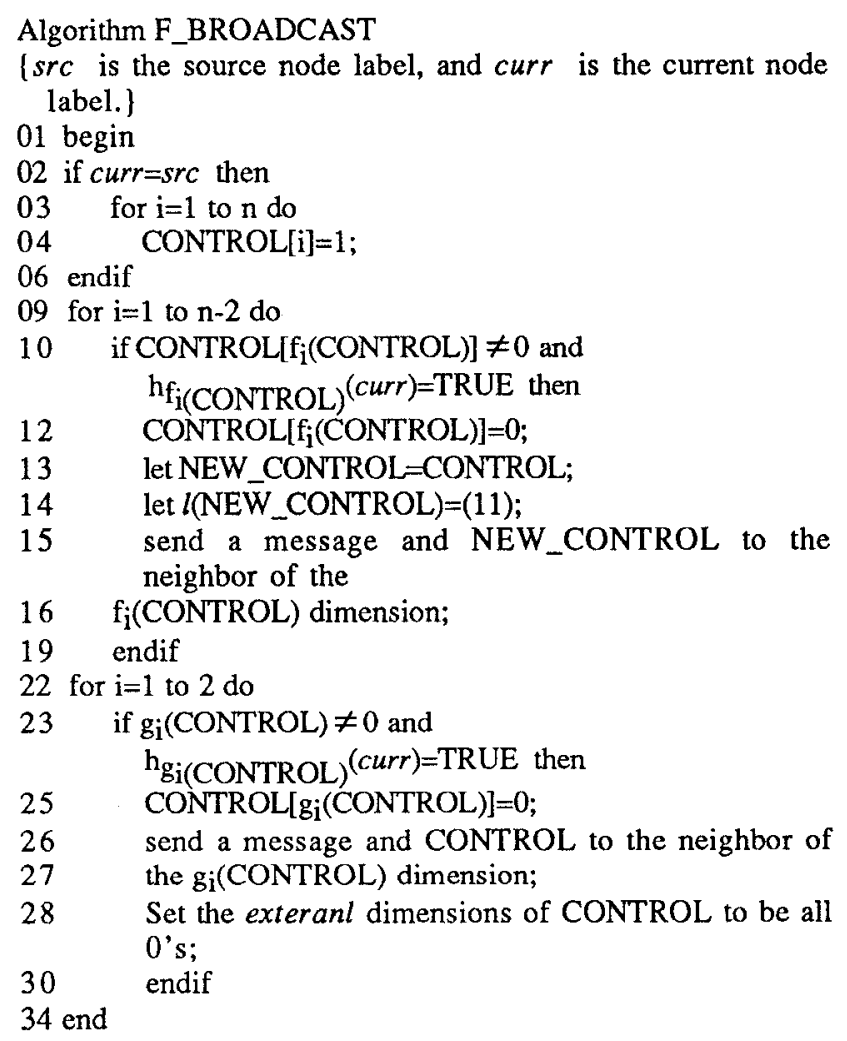

Fig. 10. A broadcasting algorithm for a faulty $n$-cube with at most $n-1$ faults.

\subsection{Comparisons}

We will examine three alternative schemes in this section. Y. Lan [12] proposed a multicasting scheme MUTICAST(source address $U_{S}$, destination list $D$ ) for a faulty $n$-cube. If we let the size of parameter $D$ in MUTICAST be 1, we can view it as a fault-tolerant routing scheme. This scheme is denoted by $F_{-} M(1)$. Moreover, a fault-tolerant broadcasting scheme is obtained 
if the size of parameter $D$ in MULTICAST is $n-1$. Note that these $n-1$ destinations are all the nodes except the source node in an $n$-cube. This scheme is denoted by F_M $(n)$. The preprocessing time $T_{p}$ for Lan's approach is spent at step 2 and step 3 in MULTICAST[12]. The time $T_{n}$ for each node is the execution time from step 4 to step 10 in MULTICAST. J. Wu [23] also proposed a broadcasting scheme F_B for a faulty hypercube. The preprocessing work for this scheme is a splitting process. The performance analysis for these schemes and ours is shown in Table 1.

The $L_{m}$ values for schemes.F_M(1) and F_M(n) are unknown because it is difficult to analyze algorithm MULTICAST. Table 1 shows that our approaches are better than others in all measures except $L_{m}$. Algorithm F_B uses more time in preprocessing, so it can obtain better result for $L_{m}$. However, our approach also has linear order for $L_{m}$. In addition, F_B only considers link faults. If faults occur with a high probability, F_B will have a greater overhead in preprocessing than our schemes. It is clear that the worst case of $L_{m}$ occurs in our schemes with a low probability since such a faulty distribution is a rare.

\begin{tabular}{|c|c|c|c|c|c|}
\hline & F_M(1) & F_ROUTING & F_BROADCAST & $F_{-} M(\mathbf{n})$ & F_B \\
\hline function & routing & routing & broadcasting & brosdcasting & broadcasting \\
\hline$T p$ & $\alpha\left(n^{2}\right)$ & $\left.\alpha_{n}\right)$ & $O(n)$ & $O\left(n^{2}\right)$ & $\left.\alpha_{n}^{2}\right)$ \\
\hline$T_{n}$ & $\alpha_{n} 3$ & $O(n)$ & $O(n)$ & $\left.O_{(n}{ }^{2}\right)$ & $O(n)$ \\
\hline$L m$ & - & $2 H(u, v)+2$ & $2 n-2$ & - & $n+2$ \\
\hline$F k$ & link/node & link/node & link/node & link/node & link \\
\hline$M s$ & $\begin{array}{l}\text { n-bit source } \\
\text { n-bit } \\
\text { destination, } \\
\text { and data } \\
\text { filed }\end{array}$ & $\begin{array}{l}\text { n-bit } \\
\text { destination, } \\
\text { and data } \\
\text { filed }\end{array}$ & $\begin{array}{l}\text { n-bit control } \\
\text { and data filed }\end{array}$ & $\begin{array}{l}\text { n-bit source, } \\
\text { n-bit } \\
\text { destination } \\
\text { and data filed }\end{array}$ & $\begin{array}{l}\text { n-bit control } \\
\text { field and } \\
\text { data filed }\end{array}$ \\
\hline$R f$ & $\mathrm{n}-1$ & $\mathrm{n}-1$ & $n-1$ & $\mathrm{n}-1$ & $n-1$ \\
\hline
\end{tabular}

Table 1. Performance analysis for several schemes.

\section{Conclusions}

In this paper, we have presented several approaches to implement fault tolerant hyperbus and hypercube multiprocessors. These approaches are based on the partitioning scheme. The partitioning scheme makes the analysis and implementation of distributed algorithms under multiple faults much easier. We have proposed a way to design a hyperbus structure in which the number of faulty processors is in a tolerant range with insignificant slowdowns. We have also presented ways for message routing and broadcasting in a faulty hypercube with at most $n$-1 faults. Compared with other existing algorithms, our methods have better performance in most measures.

\section{References}

[1] J. Bruck, R. Cypher, and D. Soroker, "Tolerating faults in hypercubes using subcube paritioning," IEEE Tran. Comput., vol. 41, no. 5, pp. 599-605, May 1992.

[2] H. Sullivan, T. Bashkow, and D. Klappholz, "A large scale, homogeneous, fully distributed parallel machine," in Proc. 4th Symp. Comput. Architecture, pp. 105-124, Mar. 1977.

[3] Y. Saad and M. H. Schultz, "Topological properties of hypercubes," IEEE Tran. Comput. vol. 37, no. 7, July 1988.

[4] A. Y. Wu, "Embedding of tree networks in to hypercubes," J. Parallel Distributed Comput., vol. 2, pp. 238-249, 1985.

[5] L. N. Bhuyan and D. P. Agrawal, "Generalized hypercube and hyperbus structures for a computer network," IEEE Tran. Comput. vol. c-33, no. 4, pp. 323-333, April 1984.

[6] M, Y. Chan and S. J. Lee, "Fault-tolerant permutation routing in hypercubes," Univ. Texas at Dallas Tech. Rep., UTDCS-5-90.

[7] J. Hastad, T. Leighton, and M. Newman, "Fast computation using faulty hypercubes," in Proc. 21st Annu. ACM Symp. Theory Comput., pp. 251-263, 1989.

[8] J. P. Sheu, Y. S. Chen, and C. Y. Chang, "Faulttolerant sorting algorithm on hypercube multicomputers," J. Parallel and Distributed Comput., pp. 185-197, 1992.

[9] T. C. Lee and J. P. Hayes, "A fault-tolerant communication scheme for hypercube computers," IEEE Tran. Comput., vol 41, no. 10, pp. 1242-1256, Oct. 1992.

[10] S. C. Chau and A. L. Liestman, "A proposal for a faulttolerance binary hypercube architecture," in Proc. Int'l Symp. on Fault-Tolerant Computing, pp. 323-330, 1989.

[11] C.S. Raghavender, P. J. Yang, and S.B. Tien, "Free dimension- An effective approach to achieve fault tolerance in hypercubes," in Proc. Int'l. Symp. on Fault-Tolerant Computing, pp. 170-177, July 1992.

[12] Y. Lan, "Multicast in faulty hypercubes," in Proc. Int'l Conf. on parallel Processing, pp. I58-61, Aug. 1992.

[13] J. Wu, "Fault-tolerant nonredundant broadcasting in hypercubes," in Proc. Int'l Conf. on Parallel Processing, pp. III23-26, Aug. 1992.

[14] M. S. Chen and K. G. Shin, "Depth-first search approach for fault-tolerant routing in hypercube multiprocessors," IEEE Tran. on Parallel and Distributed Systems, pp. 152-159, April 1990.

[15] M. Peercy and P. Banerjee, "Optimal distributed deadlock-free algorithms for routing and broadcasting in arbitrarily faulty hypercubes," in Proc. Int'l Symp. on Fault-Tolerant Computing, 1990.

[16] P. Ramanathan and K. G. Shin. "Reliable broadcast in Hypercube Multicomputers," IEEE Tran. Comput., pp. 1654-1657, Dec. 1988. 